\title{
Antithrombotic dlrugs with adjuvant action against COVID-19
}

\author{
Pierpaolo Di Micco, ${ }^{1}$ Gianluca Di Micco, ${ }^{2}$ Vincenzo Russo, ${ }^{3}$ Andrea Fontanella ${ }^{1}$ \\ ${ }^{1}$ Internal Medicine Department, Emergency Room Unit, Fatebenefratelli Hospital, Naples; ${ }^{2}$ Diagnostic Center Varelli, Naples; \\ ${ }^{3}$ Department of Translational Medical Sciences, University of Campania Luigi Vanvitelli - Monaldi Hospital, Naples, Italy
}

\begin{abstract}
Waiting for the vaccine and/or the best antiviral treatment for coronavirus infection disease 2019 (COVID-19), after its outbreak in China at the beginning of 2020 and its viral diffusion around the world in the following weeks, several drugs have been suggested for their potential adjuvant support against infection. Several drugs have been suggested to have a potential ancillary antiviral role. Circulating proteins, in particular proteases and peptidases regulated by drug functions, may interact with well-known drugs like anticoagulants, antihypertensives, antiserotoninergics, and so also with viral proteases. We here report a brief list of these drugs (i.e., heparinoids, flavonoids, antiplatelets, anticoagulants) that may interact with COVID-19.
\end{abstract}

\section{Introduction}

Venous thromboembolism (VTE) has been frequently reported as a clinical complication of hospitalization of patients affected by coronavirus infection disease 2019 (COVID-19) in particular in Intensive Care Unit. ${ }^{1,2}$ Moreover, after the viral diffusion of severe acute respiratory syndrome coronavirus 2 (SARS-CoV-2) from China to other countries in the world, several reports testified that alteration of he-

Correspondence: Pierpaolo Di Micco, Internal Medicine Department, Emergency Room Unit, Fatebenefratelli Hospital, via A. Manzoni 220, 80123 Naples, Italy.

Tel./Fax: +39.081.5981121

E-mail: pdimicco@libero.it

Key words: COVID-19; flavonoids; heparin; anticoagulants; fondaparinux.

Contributions: PDM gave his contribution in writing; GDM, gave his contribution to background and references evaluation; $\mathrm{VR}$ and $\mathrm{AF}$ gave their contribution to the revision of the draft.

Conflict of interests: the authors declare no potential conflict of interests.

Ethical approval and consent to the publication: the authors have the agreement by their Institutions to publish clinical experiences on scientific journals.

Received for publication: 28 July 2020.

Revision received: 29 August 2020.

Accepted for publication: 31 August 2020.

This work is licensed under a Creative Commons Attribution NonCommercial 4.0 License (CC BY-NC 4.0).

${ }^{\circ}$ Copyright: the Author(s), 2020

Licensee PAGEPress, Italy

Italian Journal of Medicine 2020; 14:241-244

doi:10.4081/itjm.2020.1348 mostasis as the increase of fibrinogen and D-dimer might be associated with poor outcome not only of COVID-19 per se but also of the associated VTE. ${ }^{3-5}$

For this reason, the benefit of antithrombotic drugs during COVID-19 was discovered. ${ }^{6}$ Antithrombotic drugs, in fact, may have specific antiviral and anti-inflammatory actions as testified in vitro besides their attested antithrombotic actions.

So, antithrombotics may interact with infections and with their most common complications (i.e. VTE). Heparinoids, may exert their actions because of their ability to interact with viral proteases ${ }^{7}$ and because of their antithrombotic actions. ${ }^{6}$ However, other anticoagulants can exert their action, only preventing thrombotic complications as well as aspirin and other antithrombotic drugs.

So in this report, we summarized the action of several drugs able to improve the outcome of COVID-19 infection in different ways.

\section{Flavonoids}

Flavonoids are natural products; they have a polyphenolic structure widely found in fruits and vegetables. ${ }^{8}$ They have various biochemical and antioxidant effects that may be useful during various diseases such as cancer, Alzheimer's disease, and atherosclerosis. ${ }^{9,10}$

Intriguingly, some flavonoids also have antiviral activity, as demonstrated in vitro. Their main antiviral action is related to the ability to inhibit viral proteases, in particular SARS-CoV 3-C-Like-protease. ${ }^{9} 10$

Furthermore, they may exert their antiviral action because of the affinity with oligosaccharides of the external wall of several cells that are responsible for the interaction between virus and host, in particular, such fucosylated oligosaccharides. 


\section{Heparan sulfate}

Heparan sulfate (HS) is an abundant cell-surface glycosaminoglycan (GAG) that is also present in the extracellular matrix and can bind several viruses; ${ }^{11}$ however, other GAGs present on cellular surfaces as chondroitin sulfate showed similar properties.

From a pathophysiological point of view, HS is also abundant in the respiratory tract, and it plays a role as a binding factor for coronaviruses with tropism to bronchitis and other respiratory infections. ${ }^{11}$

These actions may explain, in part, the extended tropism of this virus for cells of the respiratory tract. ${ }^{12,13}$

Moreover, HS is also present on the surface of endothelial cells, in particular, next to alveolar-capillary areas, so the capacity of SARS-CoV-2 to induce damages to lungs is due also to this property. ${ }^{11}$

Therefore, viral actions and viral capacity of SARSCoV-2 to bind host cells may slow down in the presence of high doses of HS, and these are really important for the pathophysiology of COVID-19 infection.

\section{Heparins}

Unfractionated heparins and low molecular weight heparins have a similar structure with HS, so an action similar to HS may be hypothesized during infection of coronaviruses.

Furthermore, heparins can exert their activity, increasing the action of several anti-proteases as antithrombin ${ }^{12,14}$ and other serpin family members.

Moreover, heparins and HS are also able to interact not only with clotting factors but also with endothelial cells, ${ }^{15}$ so their role in the pathophysiology of microvascular thrombosis that is described in COVID19 infection is particularly important. ${ }^{16}$

In this field, in fact, as a hypercoagulable state has been testified in several articles in patients with COVID-19 infection, ${ }^{3-5}$ pulmonary embolism and other types of thromboses may be associated with morbidity and mortality of these patients. ${ }^{1,2}$ So, heparins also assume an important role also for the thromboprophylaxis and prevention of venous thromboembolism in this infection.

For this reason, prophylactic anticoagulation with LMWH as early as possible is suggested for patients affected by COVID-19 to prevent thrombotic events and organ damage. This recommendation has been recently published in a preliminary International Society on Thrombosis and Haemostasis (ISTH).${ }^{17}$ Furthermore, after several clinical round tables, also the use of intermediated doses of LMWH has been recently reported as safe and effective in a clinical study. ${ }^{18} \mathrm{Of}$ course, patients with anticoagulant contraindications should not do it.

\section{Fondaparinux}

Fondaparinux is the synthetic pentasaccharide sequence with antithrombotic actions present in all heparins. In daily clinical practice, fondaparinux and enoxaparin have the same clinical indication for the prevention of VTE. Nevertheless, although the action of heparins toward SARS-CoV-2 has been found in vitro and in vivo, data concerning fondaparinux lack in the Literature. Italian Authors recently suggested a good antithrombotic action of fondaparinux in VTE prevention in COVID-19 in the FONDENOXAVID study (data not published).

\section{Aspirin and other antiplatelets}

A specific antiviral action of aspirin toward SARS$\mathrm{CoV}-2$ has been found neither in vitro nor in vivo, but the use of aspirin for patients with COVID-19 seems to have several advantages.

The majority of patients admitted to hospital for COVID-19 in the world, in fact, showed age older than $50 \mathrm{yy}$, and for this reason, they were already taking cardiovascular and antithrombotic drugs for the secondary prevention of cardiovascular diseases. ${ }^{19,20}$

In particular, an Italian cohort of patients admitted to the hospital for COVID-19 were on treatment with antiplatelets for secondary prevention of atherothrombotic disease (i.e., acute myocardial infarction or ischaemic stroke). Interestingly, in this study, the rate of admitted patients for COVID-19 ongoing antiplatelets was higher than the one of patients taking anticoagulants. Furthermore, in this cohort of patients, aspirin was the most frequent antiplatelet drug, while dual antiplatelet therapy was present for a minority of patients. Interestingly, patients taking aspirin during hospitalization did not show increased mortality for thrombotic disorders. ${ }^{21}$

So, based on the data of this Italian study, the suggestion not to withdraw the chronic use of aspirin has been made; the benefit of its action was based on the prevention of ischemic stroke and acute coronary syndrome, and also on the safety for the low rate of bleeding complications.

Furthermore, the safety of aspirin during the COVID-19 outbreak has also been reported in pregnant women affected by this viral infection.

\section{Warfarin and direct oral anticoagulants}

A specific action of oral anticoagulants toward SARS-CoV-2 is not present. However, for the frequent association of chronic arrhythmias or paroxysmal arrhythmias as atrial fibrillation, the use of anti-vitamin $\mathrm{K}$ (AVK) or direct oral anticoagulants (DOACs) has been found safe during the COVID-19 outbreak. $^{21,22}$ 
Furthermore, the use of AVK or DOACs is also associated with a reduced risk of developing VTE during COVID-19. ${ }^{22}$

Nevertheless, in this clinical setting, the administration of DOACs to these patients should be thoroughly performed because a possible drug-drug interaction between DOACs and antivirals. ${ }^{23}$

Furthermore, the use of any type of anticoagulant therapy is not associated with developing ARDS or death during hospitalization for COVID-19. ${ }^{22}$

So, the use of any oral anticoagulants (i.e., AVK or DOACs) may help escape different types of embolism during COVID-19, as venous thromboembolism due to hypercoagulable state andlor cardioembolism due to the frequent comorbidities as atrial fibrillation or other cardiovascular diseases. ${ }^{22}$

\section{Bleedings}

Although COVID-19 has been associated with thrombophilia and thrombosis since first reports, recently, a clinical study underlines also a relevant rate of bleedings. In this study, the incidence of thrombotic complications was similar to the rate of major bleedings. ${ }^{24}$

These data seem to be different from those previously published from other Authors.

In the Italian cohort of patients affected by COVID19 and treated with antiplatelets, the number of bleedings was inconsistent. ${ }^{21}$ Also, a clinical registry from Austria on gastrointestinal bleedings reported a reduced rate of bleeding events during the outbreak, probably also related to home rest for lockdown. ${ }^{25}$ So, because the international scientific societies have recommended prophylactic LMWH without an excess of bleedings at standard doses, and at intermediate doses, also higher doses can be considered, especially since bleeding events in COVID-19 are rare. ${ }^{26}$

Of course, bleedings may occur in such patients and could be explained by the presence of comorbidities with the trend to bleed of affected patients and also by the use of antithrombotic drugs.

So, a clinical evaluation of bleeding risk in patients affected by COVID-19 should always be considered when the administration of antithrombotic is ongoing as well as for other acute medical illnesses.

\section{Conclusions}

Several drugs have been suggested to improve the outcome of COVID-19 infection, particularly anticoagulants, for the associated risk to develop VTE during infection. This association is one of the reasons for which this outbreak has been more aggressive than the previous coronavirus epidemics (i.e., SARS and
MERS). The target of these drugs may be viral proteases or other proteases that may interfere with the clotting system or inflammatory system. Furthermore, because cardiovascular diseases with a trend to cardioembolism have been frequently reported in all populations of infected patients, also other anticoagulants as AVK or DOACs showed their useful actions during the COVID-19 outbreak.

Furthermore, additional useful actions have been demonstrated by flavonoids and heparins because of their ability to counteract the viral action on the cellular surface and the extracellular matrix. In conclusion, waiting for specific studies that may help understand the real power of these drugs in the prevention of mortality of patients with COVID-19, we strongly encourage to use the better antithrombotic strategy to prevent any thrombotic complication during COVID- 19 .

\section{References}

1. Klok FA, Kruip MJHA, van der Meer NJM, et al. Incidence of thrombotic complications in critically ill ICU patients with COVID-19. Thromb Res 2020;191: 145-14.

2. Lodigiani C, Iapichino G, Carenzo L, et al. Venous and arterial thromboembolic complications in COVID-19 patients admitted to an academic hospital in Milan, Italy. Thromb Res 2020;191:9-14.

3. Di Micco P, Russo V, Carannante N, et al. Clotting factors in COVID-19: epidemiological association and prognostic values in different clinical presentations in an Italian Cohort. J Clin Med 2020;9:E1371.

4. Tang N, Li D, Wang X, Sun Z. Abnormal coagulation parameters are associated with poor prognosis in patients with novel coronavirus pneumonia. J Thromb Haemost 2020;18:844-7.

5. Zhou F, Yu T, Du R, et al. Clinical course and risk factors for mortality of adult inpatients with COVID-19 in Wuhan, China: a retrospective cohort study [published correction appears in Lancet 2020;395:1054-62.

6. Spiezia L, Boscolo A, Poletto F, et al. COVID-19-related severe hypercoagulability in patients admitted to Intensive Care Unit for Acute Respiratory Failure. Thromb Haemost 2020;120:998-1000.

7. Lang J, Yang N, Deng J, et al. Inhibition of SARS pseudovirus cell entry by lactoferrin binding to heparansulfate proteoglycans. PLoS One 2011;6:e23710.

8. Wei J, Zhang Y, Li D, et al. Integrating network pharmacology and component analysis study on anti-atherosclerotic mechanisms of total flavonoids of Engelhardiarox burghiana leaves in mice. Chem Biodivers 2020;17:e1900629.

9. Jo S, Kim H, Kim S, et al. Characteristics of flavonoids as potent MERS-CoV 3C-like protease inhibitors. Chem Biol Drug Des 2019;94:2023-30.

10. Jo S, Kim S, Shin DH, Kim MS. Inhibition of SARSCoV 3CL protease by flavonoids. J Enzyme Inhib Med Chem 2020;35:145-51.

11. Madu IG, Chu VC, Lee H, et al. Heparansulfate is a selective attachment factor for the avian coronavirus in- 
fectious bronchitis virus Beaudette. Avian Dis 2007;51: 45-51.

12. Milewska A, Nowak P, Owczarek K, et al. Entry of human coronavirus NL63 into the cell. J Virol 2018; 92:pii:e01933-17.

13. Buczek-Thomas JA, Rich CB, Nugent MA. Hypoxia induced heparansulfate primes the extracellular matrix for endothelial cell recruitment by facilitating VEGF-fibronectin interactions. Int J Mol Sci 2019;20:pii:E5065.

14. Ghiselli G. Heparin binding proteins as therapeutic target: an historical account and current trends. Medicines (Basel) 2019;6:pii:E80.

15. Newall F. Anti-factor Xa (anti-Xa) assay. Methods Mol Biol 2013;992:265-72.

16. Thachil J. The versatile heparin in COVID-19. J Thromb Haemost 2020;18:1020-22.

17. Rauch B. Cost-effectiveness of rivaroxaban plus aspirin (dual pathway inhibition) for prevention of ischaemic events in patients with cardiovascular disease: on top optimisation of secondary prevention medication in the context of COVID-19 pandemia. Eur J Prev Cardiol 2020 Apr 17 [Epub ahead of print].

18. Mattioli M, Benfaremo D, Mancini M, et al. Safety of intermediate dose of low molecular weight heparin in COVID-19 patients. J Thromb Thrombolysis 2020 Aug 13; 1-7. [Epub ahead of print, doi: 10.1007/s11239-02002243-z].

19. Valderrama EV, Humbert K, Lord A, et al. Severe acute respiratory syndrome coronavirus 2 infection and is- chemic stroke. Stroke 2020 May 12 [Epub ahead of print, doi: STROKE AHA120030153].

20. Russo V, Di Maio M, Attena E, et al. Clinical impact of pre-admission anti-thrombotic therapy in hospitalized patients with COVID19: a multicenter observational study. Pharmacol Res 2020 [In press].

21. Thachil J, Tang N, Gando S, et al. ISTH interim guidance on recognition and management of coagulopathy in COVID-19. J Thromb Haemost 2020;18:1023-6.

22. Kow CS, Sunter W, Bain A, et al. Management of outpatient warfarin therapy amid COVID-19 pandemic: a practical guide. Am J Cardiovasc Drugs 2020 May 26; 1-9 [Epub ahead of print].

23. Vranckx P, Valgimigli M, Heidbuchel H. The Significance of drug-drug and drug-food interactions of oral anticoagulation. Arrhythm Electrophysiol Rev 2018;7: 55-61.

24. Al-Samkari H, Karp Leaf RS, Dzik WH, et al. COVID19 and coagulation: bleeding and thrombotic manifestations of SARS-CoV-2 infection. Blood 2020;136: 489-500.

25. Schmiderer A, Schwaighofer H, Niederreiter L, et al. Decline in acute upper gastrointestinal bleeding during Covid-19 pandemic after lockdown in Austria. Endoscopy 2020 May 14 [Epub ahead of print, doi: 10.1055/a-1178-4656].

26. Miesbach W, Makris M. COVID-19: coagulopathy, risk of thrombosis, and the rationale for anticoagulation. Clin Appl Thromb Hemost 2020;26:1076029620938149. 